\title{
Saúde mental em uma unidade de atenção primária à saúde: relato de experiência
}

\author{
Mental health in a healthcare primary unit: experience report \\ Monalisa Claudia Maria da Silvaa, Luiza Vieira Ferreirab ${ }^{b}$ Mariana Galvão Pereirab \\ a Enfermeira. Mestre em Enfermagem pela Universidade Federal de Juiz de Fora (UFJF). Docente do Departamento de Enfermagem Básica da \\ Faculdade de Enfermagem da UFJF, Juiz de Fora, MG, Brasil. \\ b Enfermeira. Mestranda do Programa de Pós-Graduação em Enfermagem da UFJF, Juiz de Fora, MG, Brasil.
}

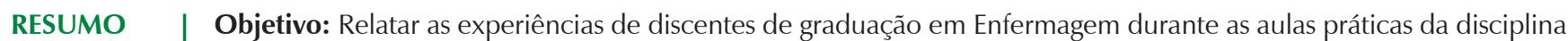
Enfermagem em Saúde Mental em uma Unidade Básica de Saúde (UBS).

Descrição da experiência: Vivência de discentes e docentes do curso de Graduação em Enfermagem da Universidade Federal de Juiz de Fora durante a realização da disciplina de Saúde Mental, em relação à implantação de um campo de prática da referida disciplina em uma UBS da cidade. A finalidade deste campo foi proporcionar uma maior visibilidade à saúde mental, com um olhar diferenciado aos usuários da Atenção Primária à Saúde (APS).

Conclusão: A inovação proposta com esta vivência na prática de saúde mental possibilitou o crescimento de todos os envolvidos no processo. A APS permite a atuação na prevenção de agravos que podem ser ocasionados pela falta de identificação e tratamento precoce de transtornos mentais. No entanto, para a construção de rotinas de ação conjunta das equipes para o desenvolvimento da saúde mental, diversas barreiras precisam ser transpostas, entre elas as dificuldades dos profissionais para a assistência aos portadores de transtorno mental dentro da Estratégia da Saúde da Família (ESF).

Palavras-chave: educação em enfermagem; saúde mental; atenção primária à saúde; estratégia saúde da família. in a Basic Health Unit (BHU).

Description of experience: Experience of students and teachers of the Nursing Graduation Course of the Federal University of Juiz de Fora during the course of the discipline of Mental Health, focusing on the implantation of a practice field of the discipline in a BHU. The purpose of this field was to provide a greater visibility to mental health, with a different look to the users of BHU.

Conclusion: The innovation proposed with this experience in mental health practice enabled the growth of all actors involved in the process. The BHU allows for actions in the prevention of diseases that can be caused by the lack of early identification and treatment of mental disorders. However, for the construction of joint action routines of teams for the development of mental health, several barriers must be overcome, including the difficulties of professionals to assist the mentally ill within the Family Health Strategy.

Keywords: education nursing; mental health; primary health care; family health strategy.

\section{Correspondência:}

MONALISA CLAudia MARIA DA SIIVA

Faculdade de Enfermagem da Universidade Federal de Juiz de Fora

Rua José Lourenço Kelmer, s/n. - Campus Universitário - São Pedro

36036-900 Juiz de Fora, MG, Brasil

E-mail: monalisacms13@gmail.com 


\section{INTRODUÇÃO}

Para iniciar a abordagem sobre a saúde mental na Atenção Primária à Saúde (APS), é imprescindível citar a contribuição das mudanças que se iniciaram no ano de 1978, que habitualmente são identificadas como o início efetivo do movimento social pelos direitos dos pacientes psiquiátricos em nosso país. A Reforma Psiquiátrica surge em consonância com os princípios de equidade e acessibilidade que regem o Sistema Único de Saúde (SUS) ${ }^{1}$.

Em 1987, temos a implementação do primeiro Centro de Apoio Psicossocial (CAPS) no Brasil, no município de São Paulo, e com ele, o início de uma forma de intervenção da Secretaria Municipal de Saúde de Santos em um hospital psiquiátrico, lugar de maus-tratos e mortes de pacientes. Esta forma de intervir evoluiu com uma repercussão em nível nacional, que confirmou, de forma inquestionável, a possibilidade de se construir uma rede de cuidados efetivamente substitutiva ao hospital psiquiátrico².

A partir de 1992, entram em vigor no país as primeiras normas federais que regulamentam a implementação de serviços de atenção diária, fundados nas experiências dos primeiros CAPS, Núcleos de Atenção Psicossocial (NAPS) e Hospitais-dia, além das primeiras normas para fiscalização e classificação dos hospitais psiquiátricos. Entretanto, somente em 2001, após mais de uma década de tramitação no Congresso Nacional, foi sancionada a Lei Federal n. 10.216, conhecida como Lei Paulo Delgado, que redireciona a assistência em saúde mental, favorecendo a oferta de tratamento em serviços de base comunitária e dispõe sobre a proteção e os direitos das pessoas com transtornos mentais ${ }^{3}$.

Destaca-se a realização, ao final do ano de 2001, em Brasília, da III Conferência Nacional de Saúde Mental, que consolida a Reforma Psiquiátrica como política de governo, confere aos CAPS o valor estratégico para a mudança do modelo de assistência, defende a construção de uma política de saúde mental para os usuários de álcool e outras drogas e estabelece o controle social como garantia do avanço da Reforma Psiquiátrica no Brasil. É, justamente, a III Conferência Nacional de Saúde Mental, com ampla participação dos movimentos sociais, de usuários e de seus familiares, que prevê fundamentos políticos e teóricos para a política de saúde mental no Brasil ${ }^{4}$.

Os CAPS são serviços de saúde municipais, que funcionam em regime aberto, com base comunitária e oferecem atendimento diário aos portadores de transtornos mentais graves e/ou persistentes. Visam diminuir o número de internações em hospitais, bem como promover a inserção social das pessoas com transtornos mentais por meio de ações intersetoriais; regular a porta de entrada da rede de assistência em saúde mental na sua área de atuação e dar suporte à atenção à saúde mental na rede básica. Os CAPS são os pontos estratégicos da rede de atenção básica, assim como da política de saúde mental num determinado território ${ }^{4}$. Competem aos CAPS o acolhimento e a assistência às pessoas com transtornos mentais graves e persistentes, procurando preservar e fortalecer os vínculos sociais do indivíduo em seu território ${ }^{5}$.

A atenção à saúde mental no âmbito da APS é componente essencial para que o sistema de saúde funcione adequadamente. A Estratégia de Saúde da Família (ESF), por sua proximidade com as famílias e comunidades que estão inseridas no entorno das Unidades Básicas de Saúde (UBS), apresenta-se como uma ação estratégica para o enfrentamento de importantes problemas de saúde pública, como os agravos vinculados ao uso abusivo de álcool, drogas e diversas outras formas de sofrimento psíquico ${ }^{6}$.

É impreterível a articulação da saúde mental com a APS, contudo, ainda se apresentam desafios para dar conta desta importante demanda. Por esta razão, o Ministério da Saúde (MS), desde 2001, vem estimulando ativamente, nas políticas de expansão, formulação e avaliação da Atenção Primária, diretrizes que incluam a dimensão subjetiva dos usuários e os problemas mais frequentes de saúde mental ${ }^{7}$. A UBS por ser a porta de entrada preferencial do usuário no Sistema Único de Saúde (SUS) é a responsável pela assistência integral à saúde da comunidade em sua área adstrita, e isso implica incluir a assistência à saúde mental, que até hoje é relegada a segundo plano ${ }^{8}$.

O processo de formação do enfermeiro deve ser pautado, dentre várias habilidades técnicas e científicas, na empatia, comunicação, relacionamento interpessoal, escuta-ativa e demais qualidades ou aptidões que são necessárias para se desenvolver uma assistência com qualidade ao próximo ${ }^{9}$.

A disciplina de Enfermagem em Saúde Mental durante a graduação possibilita uma aproximação dos discentes com a realidade da APS e da assistência aos usuários que demandam um atendimento de saúde mental.

Nesse sentido este trabalho consiste no relato de uma experiência vivenciada por discentes do Curso de Graduação em Enfermagem de uma instituição pública durante a realização das aulas práticas da disciplina de Enfermagem em Saúde Mental em uma UBS do município.

\section{DESCRIÇÃO DA EXPERIÊNCIA}

A disciplina Enfermagem em Saúde Mental é oferecida no sétimo período do Curso da Graduação em Enfermagem da Universidade Federal de Juiz de Fora(UFJF), com carga horária teórica de 30 horas e prática de 75 horas, totalizando 105 horas. Do total de sete créditos da disciplina, cinco são 
voltados para as aulas presenciais nos campos de prática e dois são realizados por meio do recurso de Ensino a Distância (EAD) para as aulas teóricas. Estas utilizam os recursos da metodologia da EAD, sendo ministradas aulas no ambiente virtual da plataforma Modular Object-Oriented Dynamic Learning Environment - Moodle - Oficial do Núcleo de Educação a Distância/UFJF, que é uma plataforma de aprendizagem a distância baseada em software livre.

Os campos utilizados para o desenvolvimento das aulas práticas correspondem ao ambiente de um CAPS do município e do hospital universitário. E, em busca de uma nova visão de abordagem à promoção da saúde mental, foram iniciadas aulas práticas no ambiente da UBS, no período de 2010 a 2012.

Tendo como principal finalidade a quebra de um ciclo pedagógico, histórico na área da saúde, o qual valoriza, a priori, a formação em ambiente hospitalar do profissional enfermeiro e ao mesmo tempo ir de encontro ao preconizado pelas diretrizes da reforma psiquiátrica, que avalia a ESF como componente fundamental para o enfrentamento dos problemas de saúde mental existente. Faz-se necessário o desenvolvimento de atividades pedagógicas com os discentes do curso, devido à sua inclusão na comunidade e à criação de vínculo com as famílias/comunidade.

O início das atividades ocorreu após a escolha da UBS para o desenvolvimento do trabalho pela docente. A unidade selecionada está situada em um bairro da zona norte do município, por tratar-se de uma unidade de saúde com algumas particularidades, entre elas o fato de possuir extensa área de abrangência, clientela variada e a prevalência de portadores de transtorno mental e dependentes químicos, como informou a assistente social e gerente da UBS.

Inicialmente, realizamos uma reunião com a equipe de saúde da UBS, para apresentação da proposta da prática de ensino e da necessidade de realizar um trabalho em conjunto com toda a equipe.

Estabeleceu-se um planejamento, que incluía a prática de visitas domiciliares (VD) e a realização semanal de grupo terapêutico com usuários indicados pela assistente social e Agentes Comunitários de Saúde (ACS). Os participantes eram selecionados por meio de busca ativa, trazendo os indivíduos e/ou famílias com história de transtorno mental ou que vivessem em situação de sofrimento psíquico, além de considerar as famílias com vulnerabilidade ao sofrimento psíquico ou adoecimento mental.

Além disso, qualquer usuário que manifestasse interesse poderia participar, pois sabe-se que a saúde mental deve ser promovida, independentemente, de diagnóstico de transtorno. Importante salientar que os discentes e docentes também participaram da busca ativa pela área de abrangência da UBS. Com o passar dos encontros, caso fosse observada uma baixa assiduidade dos participantes, seria realizada uma VD, como estratégia para mostrar-lhes que nos importávamos com suas condições e que estávamos dispostos a ajudá-los. A partir desta estratégia, o vínculo era fortalecido, confirmado pela alta assiduidade dos participantes nos grupos após a VD.

A VD é uma prática desenvolvida no âmbito da APS como uma importante estratégia no contato com o portador de transtorno mental. Esta prática, além de possibilitar e facilitar a aproximação e contato da equipe com o usuário, permite uma visão real do contexto em que ele está inserido. Podemos citar que, durante as visitas, procurava-se estabelecer uma relação de informalidade, mas com o objetivo bem definido de levantar informações sobre o usuário para posteriormente buscar, em conjunto com a equipe de saúde e indivíduo, soluções e estratégias de enfrentamento para a sua condição.

Optou-se por desenvolver ações estratégicas em um Grupo Terapêutico por reconhecermos que a natureza humana, assim como a sociedade, dita a necessidade de uma estrutura social organizada em torno de grupos. Esta técnica é utilizada para lidar com pessoas em sofrimento mental desde a II Guerra Mundial, e, desde então, tem-se observado que este modo de terapia tem efeitos que não podem ser atingidos individualmente. Uma das possíveis razões para este fato é que os grupos tendem a simular a situação familiar, em que os líderes assumem papel de figuras parentais e os membros do grupo são vistos como irmãos. Outra característica positiva do grupo é que seus membros encontram apoio na percepção de que outros têm problemas similares aos seus e, nesta perspectiva, podem buscar estratégias de mudanças mútuas ${ }^{10}$.

Foram desenvolvidos seis grupos, durante o período de 2010 a 2012, com a participação de uma média de 4 a 6 usuários em cada grupo, sendo 45 no total. As atividades eram planejadas por 5 acadêmicos de enfermagem que eram regularmente matriculados na disciplina a cada semestre letivo, contando sempre, com a supervisão dos professores da disciplina.

Os grupos foram desenvolvidos através de técnicas de relaxamento, brincadeiras lúdicas, dinâmicas e encontros dançantes, com o objetivo de interação e criação de vínculo com os participantes e, ao mesmo tempo, estimulálos para que se sentissem livres para expor suas ideias, sentimentos, preocupações. Buscou-se ainda despertar neles a autoavaliação, ao examinarem seus comportamentos com o intuito de desenvolverem novas estratégias que fossem resolutivas para suas vidas. 


\section{DISCUSSÃO}

Pode-se apreender com o desenvolvimento de aulas práticas de saúde mental na APS a necessidade de conhecer os indivíduos que estão inseridos na comunidade como uma forma de identificar aqueles que apresentam propensão ao transtorno mental e, ainda, com um grau variado de síndromes ansiosas, depressivas ou somatoformes ${ }^{11}$. De fato, foi constatado que há necessidade da integração entre os serviços prestados à comunidade pela APS e a saúde mental ${ }^{9}$.

Ressaltamos como um desafio as dificuldades de comunicação entre a comunidade e a equipe, no sentido de identificar as pessoas que necessitam de assistência e convidálas para participar das atividades desenvolvidas. A atuação do ACS é imprescindível, no que tange à identificação dos usuários na área de abrangência da UBS. Este profissional, por residir na comunidade e participar da cultura local, tem conhecimento informal de casos suspeitos de problemas mentais, que por vezes são subnotificados.

Outro desafio foi a falta de integração da equipe, entre si e com os usuários, e a ausência da equipe durante os encontros e na própria organização dos mesmos, pois os membros desta alegavam não ter "tempo" para tal demanda. Tal fato acarretou descontinuidade das ações durante o período de férias da faculdade.

A ausência de dados e de assistência à saúde mental no nível de atenção primária, especificamente na ESF, é uma condição que decorre do processo histórico de organização da assistência médico-psiquiátrica ${ }^{12}$. Trata-se, afinal, do desafio de construir uma política pública e coletiva para a saúde mental ${ }^{13}$.

Outro fator relevante desta experiência foi perceber a importância do acolhimento, o qual inclui a assistência de forma integral e individualizada, com intuito de conhecer as reais necessidades e a resolução das demandas dos usuários que frequentam os grupos oferecidos pela UBS ${ }^{14}$.

Observamos que, no início dos encontros, os poucos usuários que compareciam mostravam-se introvertidos e receosos, mas, à medida que prosseguiam as atividades, passavam a interagir com os discentes e a docente, a partir da criação de vínculo, o que possibilitou estreitar laços de confiança e assim conhecer melhor cada participante. Fato comprovado pelo comportamento de uma usuária que havia se mostrado resistente no início dos encontros, mas que, no decorrer dos dias, solicitou que o próximo grupo fosse realizado em sua residência e também se tornou participante assídua do grupo, assim como os demais.

Houve casos de usuários que preferiam ficar calados durante vários encontros sucessivos, até o momento em que optavam por falar de sua vida, de seus problemas, cada um com sua individualidade, compartilhando com todos do grupo as suas experiências de vida. Por vezes criavam empatia com um dos discentes, quando estes passavam a realizar a consulta de enfermagem com foco na demanda trazida pelo usuário.

A demanda da população pela atenção específica neste setor foi evidenciada por solicitação por parte das famílias que tomavam conhecimento do grupo e solicitavam que fosse realizada VD em sua casa, para avaliação de pessoas cujo comportamento lhes parecia atípico.

Com pouco tempo de início das atividades, foi possível perceber a importância dos encontros para sua formação profissional dos discentes, pois notaram que as ações de saúde mental podem e devem ser realizadas no contexto das equipes da ESF, sem a necessidade de ser uma área de especialização. Os profissionais devem incorporar à sua prática cotidiana o processo de cuidado integral à saúde por meio de competências e habilidades, de cuidados básicos em saúde mental, de forma que suas intervenções passem a considerar a subjetividade e a singularidade de cada indivíduo.

Os discentes vislumbraram, ainda, a relevância de a equipe de saúde da família conhecer sua área de trabalho e, sobretudo, o vínculo existente entre esta equipe e o usuário. Eles começaram a ter um feedback positivo com os relatos dos participantes dos grupos e de seus familiares, no que diz respeito às mudanças de comportamento que passavam a ocorrer na vida destas pessoas.

E, fazendo uma avaliação do processo de trabalho durante as aulas com eles, pode-se concluir que utilizar o acolhimento, o relacionamento interpessoal com o auxílio da escuta ativa vai ao encontro dos conceitos que são preconizados na APS em consonância com a ESF. Deve-se buscar promover a saúde mental dos usuários pautada na Política de Humanização que ainda é um dos grandes desafios para a consolidação e qualificação dos serviços que compõem o SUS.

A vivência desta experiência em prática de saúde mental propiciou crescimento para todos os envolvidos: docente, discentes, equipe de saúde e comunidade. Pode-se compreender que a atuação do enfermeiro perpassa por todos os sistemas que compõem o indivíduo, e a saúde mental é uma área que não pode ser relegada a segundo plano. A enfermagem precisa manter o foco de sua prática que é centrada no cuidado integral.

Por termos essa compreensão, entendemos a experiência como exitosa, uma vez que ela possibilitou o fortalecimento da educação em saúde na formação do enfermeiro. Proporcionou o contato entre os discentes e as pessoas que possuem algum tipo de sofrimento psíquico fora das instituições, em meio ao convívio familiar de suas residências, o que valorizou o espaço da APS como protagonista do cuidado. 
A APS permite a atuação na prevenção dos agravos que podem ser ocasionados pela falta de identificação e tratamento precoce de transtornos mentais, no entanto, para a construção de rotinas de ação conjunta das equipes para o desenvolvimento da saúde mental, algumas barreiras precisam ser transpostas, entre elas as dificuldades dos profissionais para a assistência aos portadores de transtorno mental dentro da Estratégia Saúde da Família e a inversão de um modelo curativista para um que promova saúde e previna doenças.

\section{REFERÊNCIAS}

1. Amarante P. Loucos pela vida: a trajetória da reforma psiquiátrica no Brasil [livro online]. Rio de Janeiro, RJ: Ed. Fiocruz, 1995 [capturado 2016 Jun 27]. 136 p. Disponível em: https://books. google.com.br/books?hl=pt-BR\&lr=\&id=CNkXAwAAQBAJ\&oi $=$ fnd $\& p g=P A 1 \& d q=$ reforma + psiquiatrica $\&$ ots $=q 06 Q \mathrm{fbGBma} \& \mathrm{~s}$ ig $=\_$EJHusl46tfprtFlpjBG_MqcUPw

2. Lopes E. Centro de Atenção Psicossocial: desafios e perspectivas em saúde mental [monografia online]. Brasília: Universidade de Brasília; 2013 [capturado 2016 Jun 27] 31 p. Disponível em: http://bdm. unb.br/bitstream/10483/5917/1/2013_EricKleberRochaLopes.pdf

3. Vaz B. O Brasil sob a lente dos Direitos Humanos: análise do conteúdo da lei 10.216/2001 [dissertação online]. Lisboa: Universidade Nova de Lisboa; 2015 [capturado 2016 Jun 28]. 130 p. Disponível em: https://run.unl.pt/handle/10362/15213

4. Tenório F. A reforma psiquiátrica brasileira, da década de 1980 aos dias atuais: história e conceitos. Hist Cienc SaudeManguinhos. 2002;9(1):25-59. https://doi.org/10.1590/S010459702002000100003

5. Fonte EMM. Da institucionalização da loucura à reforma psiquiátrica: as sete vidas da agenda pública em saúde mental no Brasil. Estud. 2013;1(18).
6. Paulon S, Neves R, Dimenstein M. A saúde mental no contexto da estratégia saúde da família no Brasil. Psicol América Lat. 2013;25:24-42.

7. Bonfim I, Bastos E, Góis C de L, Tófoli L. Apoio matricial em saúde mental na atenção primária à saúde. Interface. 2013;17(45): 287-300. https://doi.org/10.1590/S1414-32832013005000012

8. Pinto A, Jorge $M$, Vasconcelos M. Apoio matricial como dispositivo do cuidado em saúde mental na atenção primária: olhares múltiplos e dispositivos para resolubilidade. Cien Saude Colet. 2012;17(3): 653-60. https://doi.org/10.1590/S1413-81232012000300011

9. Wenceslau LD, Ortega F, Wenceslau LD, Ortega F. Saúde mental na atenção primária e Saúde Mental Global: perspectivas internacionais e cenário brasileiro. Interface - Comun Saúde, Educ. 2015;19(55):1121-32. https://doi.org/10.1590/180757622014.1152

10. Azevedo D, Miranda F. Oficinas terapêuticas como instrumento de reabilitação psicossocial: percepção de familiares. Esc Anna Nery. 2011;15(2):339-45. https://doi.org/10.1590/S1414-8145 2011000200017

11. Skapinakis P, Bellos S, Koupidis S, Grammatikopoulos I, Theodorakis $\mathrm{PN}$, Mavreas V. Prevalence and sociodemographic associations of common mental disorders in a nationally representative sample of the general population of Greece. BMC. 2013;13:163. https://doi. org/10.1186/1471-244X-13-163

12. Junqueira M, Pillon S. A assistência em saúde mental na estratégia saúde da família: uma revisão de literatura. Rev Enferm Cent-Oeste Min. 2011;1(2):260-7.

13. Hirdes A. A reforma psiquiátrica no Brasil: uma (re) visão. Cien Saude Colet. 2009;14(1):297-305. https://doi.org/10.1590/S141381232009000100036

14. Tesser C, Neto PP, Campos G. Acolhimento e (des) medicalização social: um desafio para as equipes de saúde da família. Cien Saude Colet. 2010;15(suppl.3):3615-24. https://doi.org/10.1590/S141381232010000900036 